\title{
PET Imaging for Dynamically Monitoring Neuroinflammation in APP/PS1 Mouse Model Using $\left[{ }^{18} \mathrm{~F}\right] \mathrm{DPA} 714$
}

\begin{abstract}
Wei Hu ${ }^{1,2}$, Donghui Pan ${ }^{3 \dagger}$, Yalin Wang ${ }^{4 \dagger}$, Weiqi Bao ${ }^{1}$, Chuantao Zuo ${ }^{1}$, Yihui Guan ${ }^{1}$, Fengchun Hua ${ }^{1}$, Min Yang ${ }^{3}$ and Jun Zhao ${ }^{1,5 *}$
\end{abstract}

${ }^{1}$ PET Center, Huashan Hospital, Fudan University, Shanghai, China, ${ }^{2}$ Department of Nuclear Medicine, Affiliated Wuxi People's Hospital, Nanjing Medical University, Wuxi, China, ${ }^{3}$ Key Laboratory of Nuclear Medicine, Jiangsu Key Laboratory of Molecular Nuclear Medicine, Jiangsu Institute of Nuclear Medicine, Ministry of Health, Wuxi, China, ${ }^{4}$ State Key Lab of Medical Neurobiology, Department of Integrative Medicine and Neurobiology, Institutes of Brain Science, Shanghai Medical College, Fudan University, Shanghai, China, ${ }^{5}$ Department of Nuclear Medicine, Shanghai East Hospital, Tongji University School of Medicine, Shanghai, China

OPEN ACCESS

Edited by:

Musthafa Mohamed Essa,

Sultan Qaboos University, Oman

Reviewed by:

Mar Pacheco-Herrero, Pontificia Universidad Católica Madre y Maestra, Dominican Republic Peiying Li,

Shanghai JiaoTong University School of Medicine, China

*Correspondence:

Jun Zhao

petcenter@126.com

tThese authors have contributed equally to this work

Specialty section:

This article was submitted to

Neurodegeneration,

a section of the journal

Frontiers in Neuroscience

Received: 15 April 2020

Accepted: 10 July 2020

Published: 29 September 2020

Citation:

Hu W, Pan D, Wang Y, Bao W, Zuo C, Guan $Y$, Hua $F$, Yang $M$ and Zhao $J$ (2020) PET Imaging for Dynamically

Monitoring Neuroinflammation in APP/PS1 Mouse Model Using

$\Gamma^{18}$ F]DPA714. Front. Neurosci. 14:810. doi: 10.3389/fnins.2020.00810
Background: In the pathogenesis of Alzheimer's disease (AD), microglia play an increasingly important role. Molecular imaging of neuroinflammatory targeting microglia activation and the high expression of 18-kDa translocator protein (TSPO) has become a hot topic of research in recent years. Dynamic monitoring neuroinflammation is crucial for discovering the best time point of anti-inflammatory therapy. Motivated by this, Positron emission tomography (PET) imaging in an APP/PS1 mouse model of AD, using ${ }^{18} \mathrm{~F}$ labeled DPA-714 to monitor microglia activation and neuroinflammation, were performed in this paper.

Methods: We prepared $\left[{ }^{18} \mathrm{~F}\right] \mathrm{DPA} 714$ and tested the biological characteristics of the molecular probe in normal mice. To obtain a higher radiochemical yield, we improved the $\left[{ }^{18} \mathrm{~F}\right]$-fluorination conditions in the precursor dosage, reaction temperature, and synthesis time. We performed $\left[{ }^{18}\right.$ F]DPA714 PET scanning on APP/PS1 mice at 6-7, 9-10, 12-13, and 15-16 months of age, respectively. The same experiments were conducted in Wildtype (Wt) mice as a control. Referring to the $\left[{ }^{18} \mathrm{~F}\right] \mathrm{DPA} 714$ concentrated situation in the brain, we performed blocking experiments with PK11195 (1 mg/kg) in 12-13-months-old APP/PS1 mice to confirm the specificity of $\left[{ }^{18} \mathrm{~F}\right] \mathrm{DPA} 714$ for TSPO in the APP/PS1 mice. Reconstructed brain PET images, fused with the Magnetic Resonance Imaging (MRI) template atlas, and the volumes of interests (VOIs) of the hippocampus and cortex were determined. The distribution of [ ${ }^{18} \mathrm{~F}$ DPA714 in the brain tissues of $15-16$-months-old APP/PS1 and Wt mice were studied by immunofluorescence staining.

Results: Through the reaction of ${ }^{18} \mathrm{~F}$, with $2 \mathrm{mg}$ precursor dissolved in $300 \mathrm{ul}$ acetonitrile at $105^{\circ} \mathrm{C}$ for $10 \mathrm{~min}$, we obtained the optimal radiochemical yield of $42.3 \pm 5.1 \%$ (non-decay correction). Quantitative analysis of brain PET images showed that the $\left[{ }^{18}\right.$ FDPA714 uptake in the cortex and hippocampus of 12-13-months-old APP/PS1 mice was higher than that of the control mice of the same age (cortex/muscle: $2.77 \pm$ 0.13 vs. $1.93 \pm 0.32, p=0.0014$; hippocampus/muscle: $3.33 \pm 0.10$ vs. $2.10 \pm 0.35$, 
$p=0.0008)$. The same significant difference was found between 15- and 16months-old APP/PS1 mice (cortex/muscle: $2.64 \pm 0.14$ vs. $1.86 \pm 0.52, p=0.0159$; hippocampus/muscle: $2.89 \pm 0.53$ vs. $1.77 \pm 0.48, p=0.0050)$. Immunofluorescence staining showed that the activation of microglia and the level of TSPO expression in the cortex and hippocampus of APP/PS1 mice were significantly higher than Wt mice.

Conclusion: $\left[{ }^{18} \mathrm{~F}\right] \mathrm{DPA} 714$, a molecular probe for targeting TSPO, showed great potential in monitoring microglia activation and neuroinflammation, which can be helpful in discovering the best time point for anti-inflammatory therapy in AD.

Keywords: microglia, translocator protein (18 kDa), $\left[{ }^{18} \mathrm{~F}\right] \mathrm{DPA} 714, \mathrm{PET}$, Alzheimer's diseases, APP/PS1

\section{BACKGROUND}

Alzheimer's disease (AD) is the chronic inflammation of the central nervous system (CNS), including focal brain injury and a lot of beta-amyloid protein (A beta, $\mathrm{A} \beta$ ) deposition (Golde, 2016). In recent years, it has been found that microglia play a major role in the pathogenesis of $\mathrm{AD}$, which is also currently a hot topic in the study of neurodegenerative diseases. Microglia activated by $\mathrm{A} \beta$ depositions is one of the main mechanisms of neuroinflammation. Activated microglia produce a large number of precursors of inflammatory factors, resulting in the occurrence of neurodegenerative diseases (Politis et al., 2012; Li et al., 2014). The translocator protein (TSPO, $18 \mathrm{kDa}$ ), also known as the peripheral benzodiazepine receptor (PBR), is mainly localized in the outer mitochondrial membrane of steroid-synthesizing cells of the peripheral and central nervous system. Normally, the central nervous system is restricted to glial cells (astrocytes and microglia) which are expressed less in the CNS. The expression of TSPO in microglia is significantly increased in response to injury or inflammatory of the brain disease, so it can be used as an important neuroinflammation marker (Chen and Guilarte, 2007; Rupprecht et al., 2012).

Although a lot of research has been done, the role of activated microglia in the dynamic stages of neuroinflammation is still unclear. Positron emission tomography (PET) tracers targeting TSPO has been widely used to detect the activation of microglia in both preclinical and clinical studies (James and Gambhir, 2012). $\left[{ }^{11} \mathrm{C}\right] \mathrm{PK} 11195$ is the first tracer used in neuroinflammatory PET imaging studies. Although it has been studied for more than 20 years, $\left[{ }^{11} \mathrm{C}\right]$ PK11195 still has many limitations: the specificity of $\left[{ }^{11} \mathrm{C}\right] \mathrm{PK} 11195$ binding to TSPO is low, which affects the image quality and quantitative analysis of microglia activation; furthermore, carbon-11 has a short half-life $(20.38 \mathrm{~min})$ and requires field cyclotron for nuclide preparation, which limits its wide clinical application (Chauveau et al., 2008). In the past decade, many researchers have improved the properties of TSPO tracers and developed new specific radio-ligands, including $\left[{ }^{18} \mathrm{~F}\right] \mathrm{DPA} 714$ (Vivash et al., 2016). $\left[{ }^{18} \mathrm{~F}\right] \mathrm{DPA} 714$ is a pyrazolopyrimidine TSPO ligand with high affinity, which has a good stability in plasma and brain tissue and has been used in animal and human PET imaging studies (James et al., 2008; Arlicot et al., 2012; Peyronneau et al., 2012). Compared with $\left[{ }^{11} \mathrm{C}\right] \mathrm{PK} 11195,\left[{ }^{18} \mathrm{~F}\right] \mathrm{DPA} 714$ has a significant improvement in the affinity and decrease of nonspecific binding to TSPO (Chauveau et al., 2009). The purpose of this study is to evaluate the role of $\left[{ }^{18} \mathrm{~F}\right] \mathrm{DPA} 714$ PET imaging in monitoring the TSPO levels in the mouse model of APP/PS1 mice at different months, which can help us to better understand the action of microglia in the pathogenesis of $\mathrm{AD}$ neuroinflammation. To the best of our knowledge (Takkinen et al., 2016) is the only research that shares a similar aim to our paper. It is a longitudinal PET study which demonstrated decreased energy metabolism and increased inflammation in the brains of APP/PS1-21 mice compared to WT mice. Our work, however, differs in terms of experimental method and design: (1) The radioactive labeling condition of $\left[{ }^{18} \mathrm{~F}\right] \mathrm{DPA} 714$ is optimized in our work, which increases the radiochemical yield with fewer precursors and a shorter synthesis time; (2) In this work, PET dynamic imaging on mice were performed directly after projection, which was crucial in determining the best time point for PET imaging after injection. (3) A blocking experiment using PK11195 demonstrated that $\left[{ }^{18}\right.$ F]DPA714 had improved specificity for TSPO.

\section{METHOD}

\subsection{General Materials}

All chemicals used are of analytical grade and were purchased from Aldrich and Sigma. $\left[{ }^{18} \mathrm{~F}\right]$-F-radionuclide was obtained from Jiangsu Institute of Nuclear Medicine. We conducted mouse PET imaging on an Inveon micro-PET scanner (Siemens Medical Solutions, Germany). We received the precursor DPA714 from Professor Xiaoyuan Chen at the National Institutes of Health (NIH).

\subsection{Animals}

B6.Cg-Tg (APPswe, PSEN1dE9)85Dbo/Mmjax (APP/PS1) and $\mathrm{Wt}$ mice were purchased from Shanghai Model Organisms Center, Inc. and bred by the Jiangsu Institute of Nuclear Medicine. The provenances were obtained from the Jackson Laboratory. According to the Jackson Laboratory website's description, APP/PS1 mice could express chimeric mouse/human amyloid precursor protein (Mo/Hu APP695swe) and mutant human presenilin1 (PS1-dE9), both directed to the neurons of central nervous system, which is associated with early-onset Alzheimer's disease. The APP/PS1 mice produce 
TABLE 1 | Result of the different temperature and concentration of precursor of the $\left[{ }^{18} \mathrm{~F}\right]$-fluorination yield of $\left[{ }^{18} \mathrm{~F}\right] \mathrm{DPA} 714$ with $10 \mathrm{~min}$ reaction time (non-decay-corrected; $n=4$ ).

\begin{tabular}{lcc}
\hline Temperature $\left({ }^{\circ} \mathbf{C}\right)$ & \multicolumn{2}{c}{ Concentration of precursor $(\mathbf{m g} / \mathbf{m l})$} \\
\cline { 2 - 3 } & $\mathbf{4}$ & $\mathbf{6 . 7}$ \\
\hline 95 & $16.3 \pm 5.0 \%$ & $31.6 \pm 3.7 \%$ \\
105 & $25.4 \pm 3.8 \%$ & $42.3 \pm 5.1 \%$ \\
\hline
\end{tabular}

TABLE 2 | Result of the different reaction time on the $\left[{ }^{18} \mathrm{~F}\right]$-fluorination yield of $\left[{ }^{18} \mathrm{~F}\right] \mathrm{DPA} 714$ at $105^{\circ} \mathrm{C}$ reaction temperature (non-decay-corrected; $n=4$ ).

\begin{tabular}{lcc}
\hline Reaction time $(\mathbf{m i n})$ & \multicolumn{2}{c}{ Concentration of precursor $(\mathbf{m g} / \mathbf{m l})$} \\
\cline { 2 - 3 } & $\mathbf{4}$ & 6.7 \\
\hline 5 & $9.0 \pm 0.6 \%$ & $21.5 \pm 1.0 \%$ \\
10 & $25.4 \pm 3.8 \%$ & $42.3 \pm 5.1 \%$ \\
\hline
\end{tabular}

$\beta$-amyloid deposits in the brain by $6-7$ months of age and have elevated beta-amyloid levels between 6 and 12 months of age (Jankowsky and L., 2003; Garcia-Alloza et al., 2007; Xiong et al., 2011). APP/PS1 and Wt mice were identified by PCR assays of tail DNA. They were housed separately in an enriched environment with a 12-h light/12-h dark cycle, constant temperature (23 \pm $\left.1{ }^{\circ} \mathrm{C}\right)$, and humidity $(55 \pm 5 \%)$ with available food and water.

\subsection{Synthesis of $\left[{ }^{18} \mathrm{~F}\right] \mathrm{DPA} 714$}

Based on previous reports (James et al., 2008; Arlicot et al., 2012; Wang et al., 2014), we have improved the labeling method. ${ }^{18} \mathrm{~F}$ Fluoride was separated from ${ }^{18} \mathrm{O}-\mathrm{H}_{2} \mathrm{O}$ by QMA-Light Sep-Pak anion-exchange resin (Waters) preconditioned with $10 \mathrm{ml}$ of $0.5 \mathrm{M} \mathrm{KHCO}_{3}$ and $15 \mathrm{ml}$ deionized water. Then ${ }^{18} \mathrm{~F}$-Fluoride was eluted from Waters QMA anion exchange cartridge and transferred to the reaction vessel (Thermo Fisher Scientific, 2 $\mathrm{ml}$ ) with eluent solution containing $\mathrm{K}_{2} \mathrm{CO}_{3}(3 \mathrm{mg}$ in $10 \mathrm{ul}$ of pure water), acetonitrile (90 ul), and $15 \mathrm{mg}$ of Kryptofix [2.2.2] (Sigma, America). The solution was dried completely by azeotropic distillation with acetonitrile $(3 \times 1.0 \mathrm{ml})$ (Acros, Belgium) under the mild flow of nitrogen at $110^{\circ} \mathrm{C}$. Then, DPA$714(2 \mathrm{mg})$ dissolved in $500 \mathrm{ul}(4 \mathrm{mg} / \mathrm{ml})$ or $300 \mathrm{ul}(6.7 \mathrm{mg} / \mathrm{ml})$ acetonitrile (Acros, Belgium) was added to the dry ${ }^{18} \mathrm{~F}$-labeled K-F-Kryptofix-222 complex. The reaction mixture was heated at 95 or $105^{\circ} \mathrm{C}$ for 5 or $10 \mathrm{~min}$ and developed a brown color. After reaction, we diluted the mixture with sterile water and then separated it through a tC18 cartridge (Waters Sep-Pak Accell Light tC18 cartridge washed with $10 \mathrm{ml}$ of ethanol and $15 \mathrm{ml}$ deionized water before use). The products gathered by the tC18 cartridge were washed with deionized water $(20 \mathrm{ml})$ and then eluted with acetonitrile $(200 \mathrm{ul})$. The crude mixtures were injected into a Waters X-Terra $\mathrm{C}_{18} 10$-mm $(250 \times 10$ $\mathrm{mm}$, Phenomenex, America) semi-preparative reversed-phase high-performance liquid chromatography (HPLC) column with a mobile phase of $\mathrm{CH}_{3} \mathrm{CN}$, deionized water and triethylamine $(50: 50: 0.1, \mathrm{v} / \mathrm{v} / \mathrm{v})$ at a flow rate of $3.0 \mathrm{~mL} / \mathrm{min}$. The purified products were eluted with $0.3 \mathrm{ml}$ ethanol from tC18 cartridge and diluted with $0.9 \%$ saline to make $\left[{ }^{18} \mathrm{~F}\right] \mathrm{DPA} 714$ could be used for intravenous injection. The final solution with known volume and radioactivity were injected into a Waters X-Terra tC18 $(250 \times$ $4.6 \mathrm{~mm}$, Phenomenex, America) analytic reversed-phase HPLC column to determine its radio-chemical purity. $\left[{ }^{18} \mathrm{~F}\right] \mathrm{DPA} 714$ was eluted with a mobile phase of $\mathrm{CH}_{3} \mathrm{CN}$, deionized water and triethylamine $(40: 60: 0.1, \mathrm{v} / \mathrm{v} / \mathrm{v})$ at a flow rate of $1.0 \mathrm{~mL} / \mathrm{min}$.

\subsection{PET Scanning and Image Fusion}

The study was performed using four female and four male APP/PS1 mice (APP/PS1, $n=8$ ), and four female and four male Wt mice as the control group ( $\mathrm{Wt}, n=8$ ). These 16 mice were scanned at ages of $6-7,9-10,12-13$, and 15-16 months continuously. PET acquisitions were performed on a micro-PET (Inveon,Siemens) which had an effective axial/transaxial field of view (FOV) of $12.7 / 10 \mathrm{~cm}$. Prior to the scans, mice were anesthetized using isoflurane $(2.0-3.0 \%$ for induction and 1.5$2.5 \%$ for maintenance). They were placed into the scanner so that their brains were located in the center of the FOV. Dynamic scans $(60 \mathrm{~min}$ ) for APP/PS1 and Wt mice were performed after intravenous injection of $\left[{ }^{18} \mathrm{~F}\right] \mathrm{DPA} 714(3.7-5.5 \mathrm{MBq}, 0.2 \mathrm{ml})$. We conducted blocking studies, which pre-treated 12-13-monthsold APP/PS1 mice with PK11195 (1 mg/kg; Sigma Aldrich) $10 \mathrm{~min}$ before radioligand administration. PK11195 is a TSPO antagonist. Pre-treatment with PK11195 resulted in saturation of the TSPO receptors, blocking them for $\left[{ }^{18} \mathrm{~F}\right] \mathrm{DPA} 714$ occupancy (Keller et al., 2018). All list-mode data were reconstructed using the OSEM 2D algorithm (frames, $4 \times 30,5 \times 150,6 \times$ $450 \mathrm{~s}$ ) with no attenuation correction. In the image analysis software PMOD (3.7, PMOD Technologies, Zurich, Switzerland; www.pmod.com), the reconstructed PET measurements were manually aligned with a mice brain MRI template atlas. We selected the hippocampus and cortex as the VOIs and calculated their percentages of injected dose per gram (\%ID/g). Timeactivity curves (TACs) were calculated as a mean of the VOIs and normalized for the $\% \mathrm{ID} / \mathrm{g}$. To calculate the signal-to-background ratios, we divided the uptake of VOIs by the intake of reference region (muscle). For muscle VOIs, two regions were drawn per arm of each mouse (i.e., four muscle regions per mouse) using the PET image, and then the average \%ID/g from all four muscle VOIs for each mouse was calculated to provide the overall muscle \%ID/g (James et al., 2015).

\subsection{Immunofluorescence Staining}

The 15-16-months-old Wt and APP/PS1 mice were perfused immediately after PET imaging to take whole brain tissue samples. Through CM-1900 cryostat (Leica, Germany), we obtained mice brain cryosections with a thickness of $30 \mathrm{um}$. To quantify Iba-1 and TSPO staining, three sections per mouse in the 15-16-months-old group ( $n=8 \mathrm{Wt}$ and $n=8 \mathrm{APP} / \mathrm{PS} 1$ ) were analyzed. All the sections taken from the cryoprotectant were gently washed with 0.01 M Phosphate Buffered Saline (PBS) for $10 \mathrm{~min}$, and then we blocked the non-specific binding sites with PBS containing $1 \%$ bovine serum albumin for $1 \mathrm{~h}$. The treated slices needed to be incubated overnight with the primary antibodies at $4^{\circ} \mathrm{C}$ and then with the secondary antibodies at room 


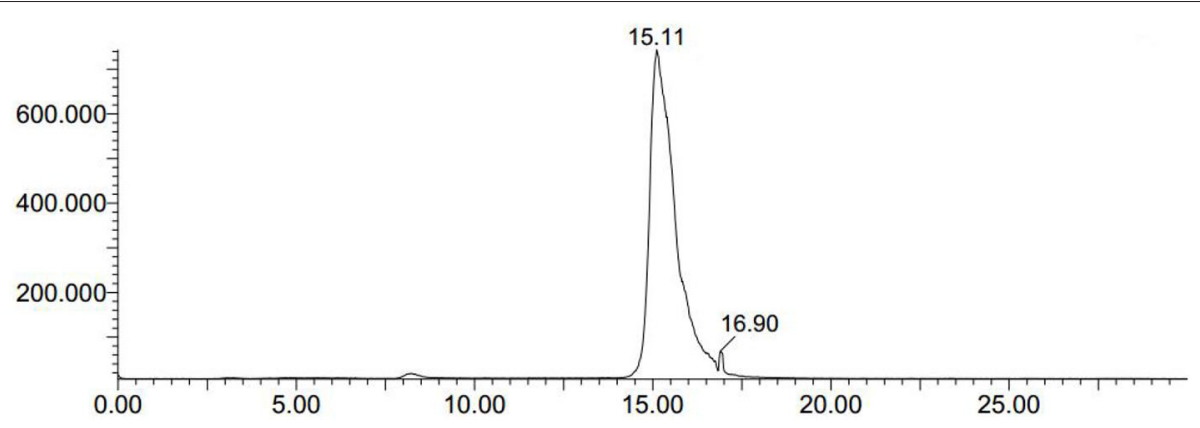

FIGURE 1 | The crude mixtures of $\left[{ }^{18} \mathrm{~F}\right] \mathrm{DPA} 714$ was extracted using semi-preparative reversed-phase HPLC. The retention time (tR) of $\left[{ }^{18} \mathrm{~F}\right] \mathrm{DPA} 714$ was 15.11 min with a mobile phase of $\mathrm{CH}_{3} \mathrm{CN}$, deionized water and triethylamine $(50: 50: 0.1, \mathrm{v} / \mathrm{v} / \mathrm{v})$, liquid pump flow rate $3.0 \mathrm{~mL} / \mathrm{min}$.

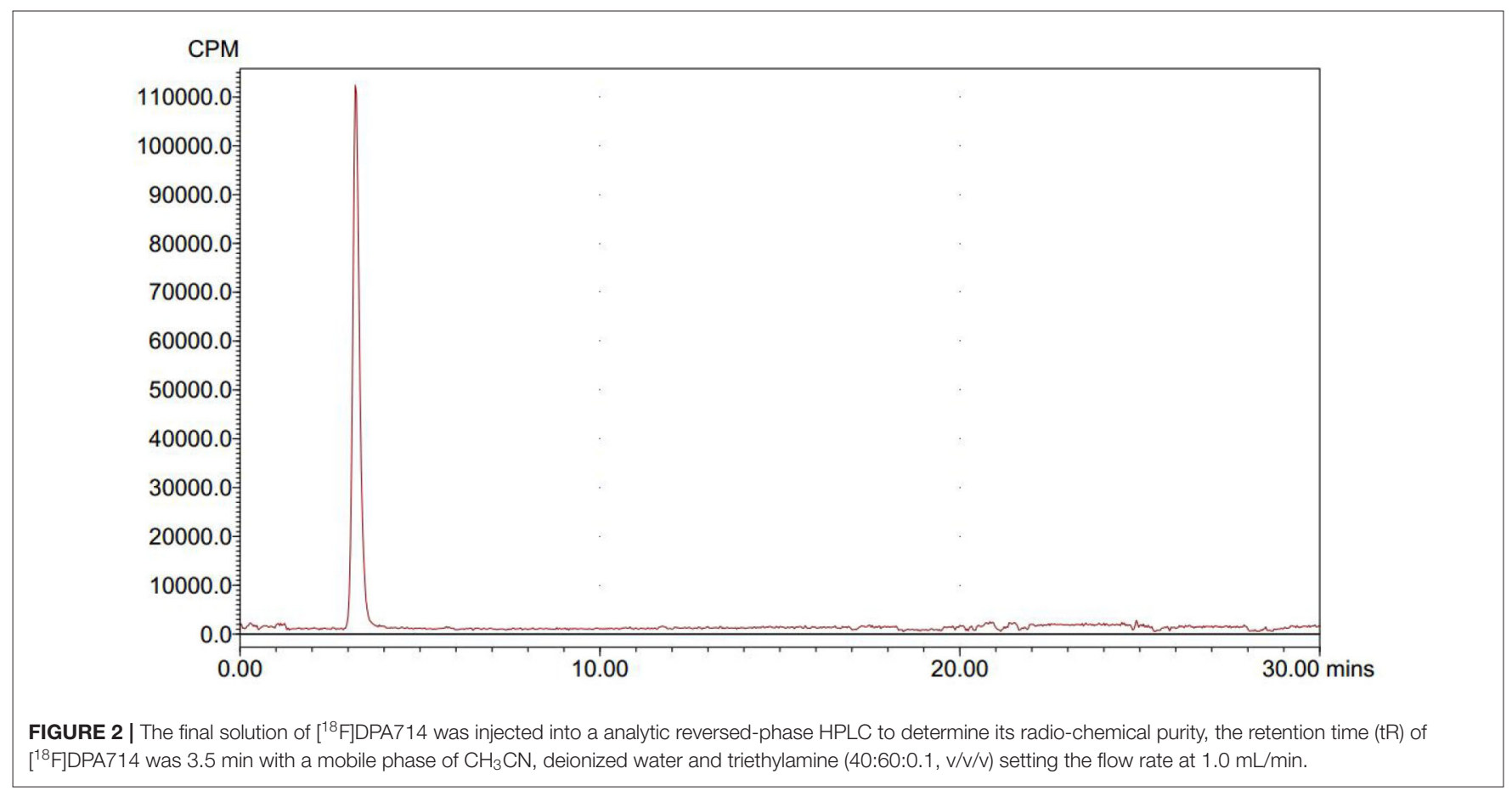

temperature for $1 \mathrm{~h}$. For different staining targets, it is critical to choose the specific antibodies and appropriate concentrations.

The antibodies and concentrations we used are as follows: goat anti-mouse Iba-1 (a microglial marker) antibody (1:500, ab5076, Abcam), rabbit anti-mouse PBR (TSPO) antibody (1:200, sc-20120, Santa Cruz); FITC-conjugated donkey antigoat antibody (1:1,000, 1608643, Life technologies), FITCconjugated donkey anti-rabbit antibody (1:1,000, 1674651, Life technologies). All tissue slices were mounted with medium containing 4,6-diamidino-2-phenylindole (DAPI, G3016-TI96, Southern Biotech) and then observed using a confocal laser scanning microscope (Leica, Germany).

One immunofluorescence image corresponds to each section of one mouse, and we have $8 \mathrm{Wt}$ mice and $8 \mathrm{APP} / \mathrm{PS} 1$ mice with three sections per mouse. Therefore, we obtained 48 immunofluorescence figures of Iba-1 and TSPO after staining. These figures were imported into Image J software (1.42q) and results were analyzed and compared. The software first calculated mean gray value (mean) of each figure, which represented the figure fluorescent intensity and can be obtained as following equation: Mean Gray Value = Integrated Density/Area. Based on the mean gray values of both Iba- 1 and TSPO, we drew the relative fluorescence intensity chart to compare them visually. Furthermore, the $T$-test was conducted to test differences for Wt and APP/PS1 mice. Figures of Dapi, Iba-1, and TSPO in the same field of vision were also merged by Image J software to observe the overlapping situation of three staining target areas.

\subsection{Statistical Analysis}

We presented all the data in the form of means \pm standard deviation (mean \pm SD). Statistical analysis was conducted using Graph-Pad Prism (version 5.1). Difference $<0.05$ was considered to be statistically significant. 
A

Cortex

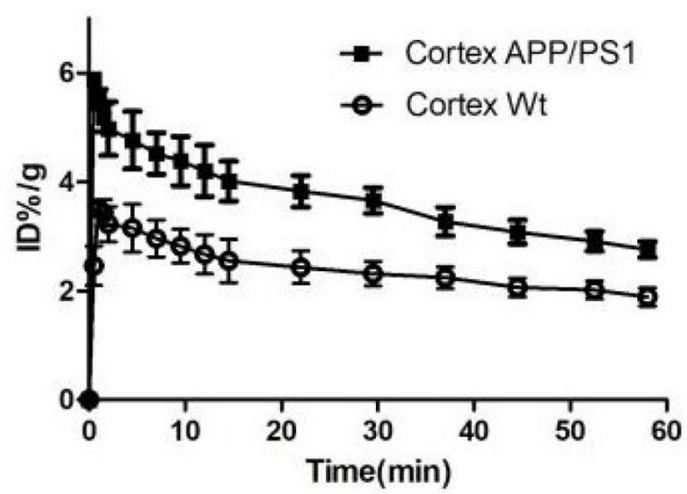

B Cortex(blocking study)

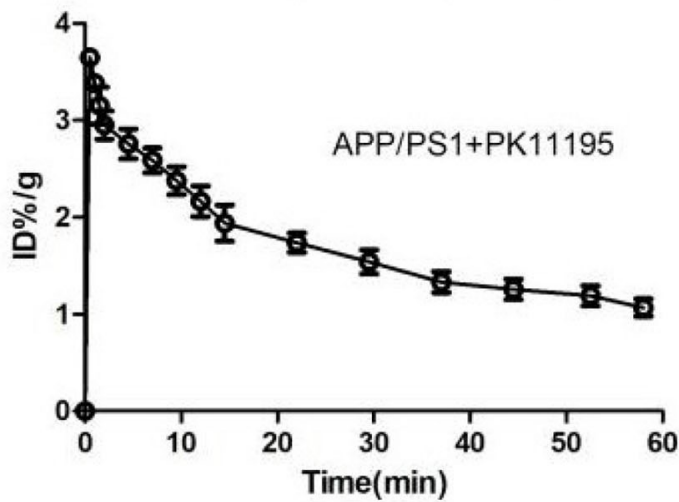

Hippocampus

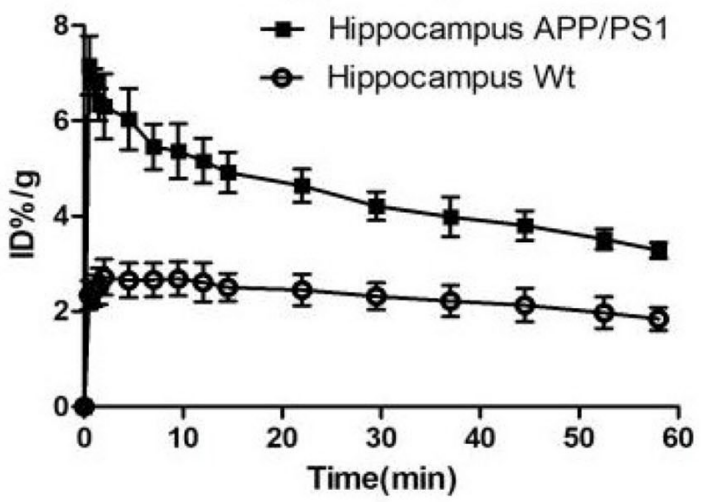

Hippocampus(blocking study)

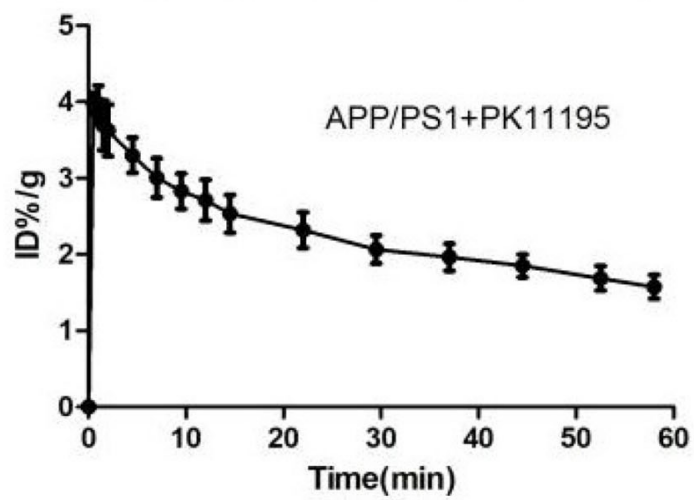

FIGURE 3 | (A) The time-activity curves (TACs) of $\left[{ }^{18}\right.$ F]DPA714 accumulation in cortex and hippocampus of 12-13-months-old APP/PS1 $(n=8)$ and Wt $(n=8)$ mice using dynamic scan (60 min). (B) In blocking studies, the time-activity curves (TACs) of $\left[{ }^{18} \mathrm{~F}\right] \mathrm{DPA} 714$ accumulation in cortex and hippocampus pre-treated 12-13-months-old APP/PS1 mice $(n=8)$ with PK11195 10 min before radioligand administration.
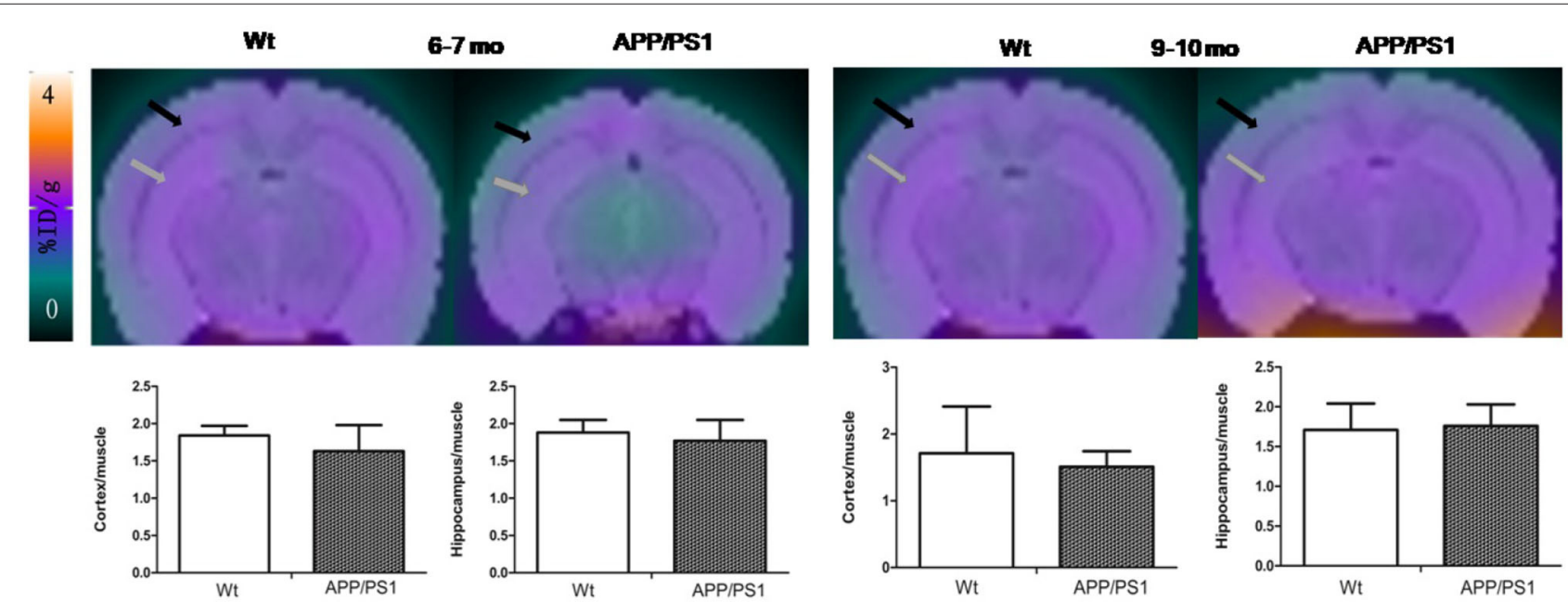

FIGURE 4 | The PET images were aligned with mice brain MRI template atlas. We selected hippocampus and cortex as the VOls and calculated their percentages of injected dose per gram (\%ID/g). PET/MRI template atlas fusion images and graphs representing $\left[^{18} \mathrm{~F}\right.$ DPA714 signals in APP/PS1 vs. Wt mice 6-7 ( $\left.n=8\right)$ and $9-10$ $(n=8)$ months of age. Black and gray arrows point to cortex and hippocampus, respectively. 

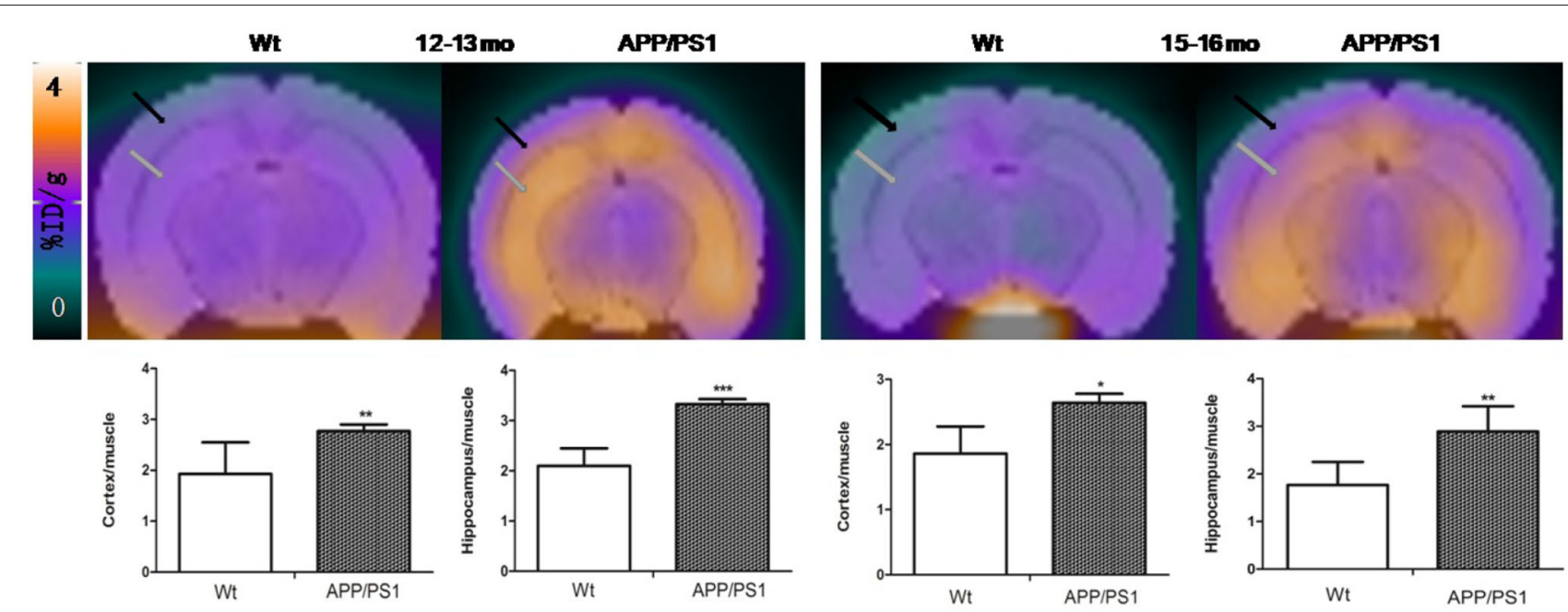

FIGURE 5 | The PET images were aligned with the mouse brain MRI template atlas. We selected the hippocampus and cortex as the VOls and calculated their percentages of injected dose per gram (\%ID/g). PET/MRI template atlas fusion images and graphs representing $\left.{ }^{18} \mathrm{~F}\right] \mathrm{DPA} 714$ signals in APP/PS1 vs. Wt mice $12-13$ and 15-16 months of age. Black and gray arrows point to cortex and hippocampus, respectively $\left({ }^{*} p<0.05,{ }^{* *} p<0.01,{ }^{* * *} p<0.001\right)$.
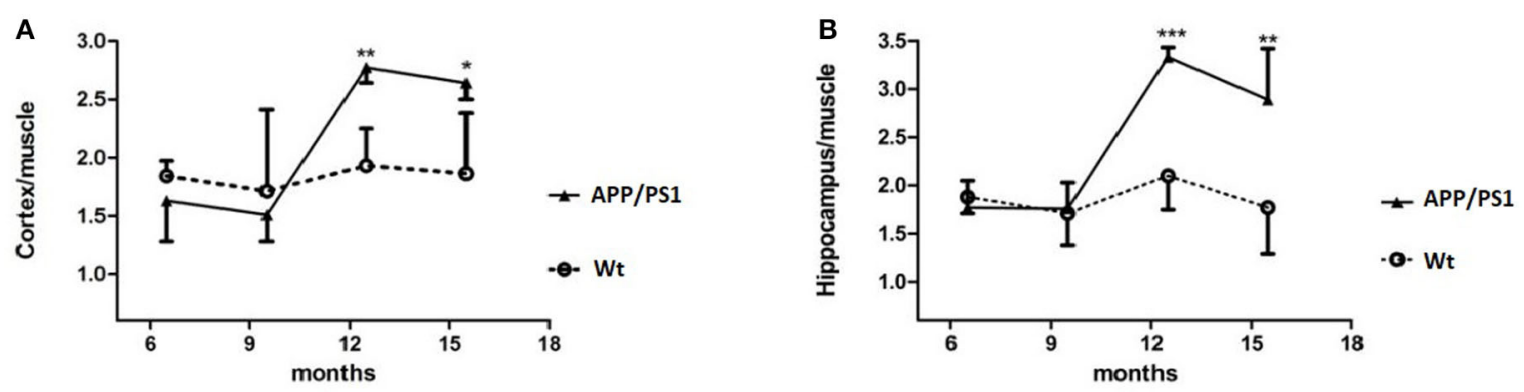

FIGURE $6 \mid \%$ ID/g to muscle of $\left[{ }^{18}\right.$ F]DPA714 accumulation in the cortex and hippocampus, at $6-7,9-10,12-13$, and $15-16$ months of age. ${ }^{*} p<0.05,{ }^{* *} p<0.01$, and ${ }^{* * *} p<0.001$ compared with Wt age-matched mice ( $T$ test).

\section{RESULTS}

\subsection{Radiochemistry}

We conducted radiolabeling of DPA-714 and ${ }^{18} \mathrm{~F}$ by nucleophilic aliphatic substitution and selected the optimal concentration, temperature and reaction time of the precursor. Through the reaction of ${ }^{18} \mathrm{~F}$ with $2 \mathrm{mg}$ precursor dissolved in $300 \mathrm{ul}$ acetonitrile at $105^{\circ} \mathrm{C}$ for $10 \mathrm{~min}$, we obtained the optimal radiochemical yield of $42.3 \pm 5.1 \%$ (nondecay correction) (Table 1). Reaction time is another important condition affecting the radiochemical yield (Table 2). With the increase of the reaction time, the rate of radiochemical yield also increased. Total radio-synthesis time for the preparation of $\left[{ }^{18} \mathrm{~F}\right] \mathrm{DPA} 714$ was $30-35 \mathrm{~min}$. The retention time (tR) of $\left[{ }^{18} \mathrm{~F}\right] \mathrm{DPA} 714$ in the semi-preparative reversed-phase HPLC system was 15.11 min (Figure 1) and the radiochemical purity of $\left[{ }^{18} \mathrm{~F}\right] \mathrm{DPA} 714$ is $99.9 \%$ (Figure 2).

\subsection{Mice PET}

We used $\left[{ }^{18} \mathrm{~F}\right] \mathrm{DPA} 714$ micro-PET imaging to monitor the TSPO levels of APP/PS1 mice in different ages and found that the microglia of different aged mice displayed different degrees of activation. Quantitative analysis of $\left[{ }^{18} \mathrm{~F}\right] \mathrm{DPA} 714$ PET images revealed that there was no significant difference in cortical and hippocampal signals between APP/PS1 and Wt mice aged 6-7 and 9-10 months. Conversely, APP/PS1 and Wt mice aged 12-13 and 15-16 months showed a significant difference, known to display substantial microglia activation. We performed $\left[{ }^{18}\right.$ F]DPA714 dynamic PET imaging for APP/PS1 and Wt mice. The time-activity curves (TACs) of dynamic imaging (Figure 3A) showed that $\left[{ }^{18} \mathrm{~F}\right] \mathrm{DPA} 714$ uptake in the cortex and hippocampus of 12-13-months-old APP/PS1 mice was significantly higher than that of Wt mice at 40-50 min post-injection (cortex: $3.08 \pm$ 0.17 vs. $2.06 \pm 0.22 \% \mathrm{ID} / \mathrm{g},{ }^{*} P<0.05$; hippocampus: $3.80 \pm 0.44$ vs. $\left.2.13 \pm 0.49 \% \mathrm{ID} / \mathrm{g},{ }^{* *} P<0.005\right)$. In the blocking experiments, 


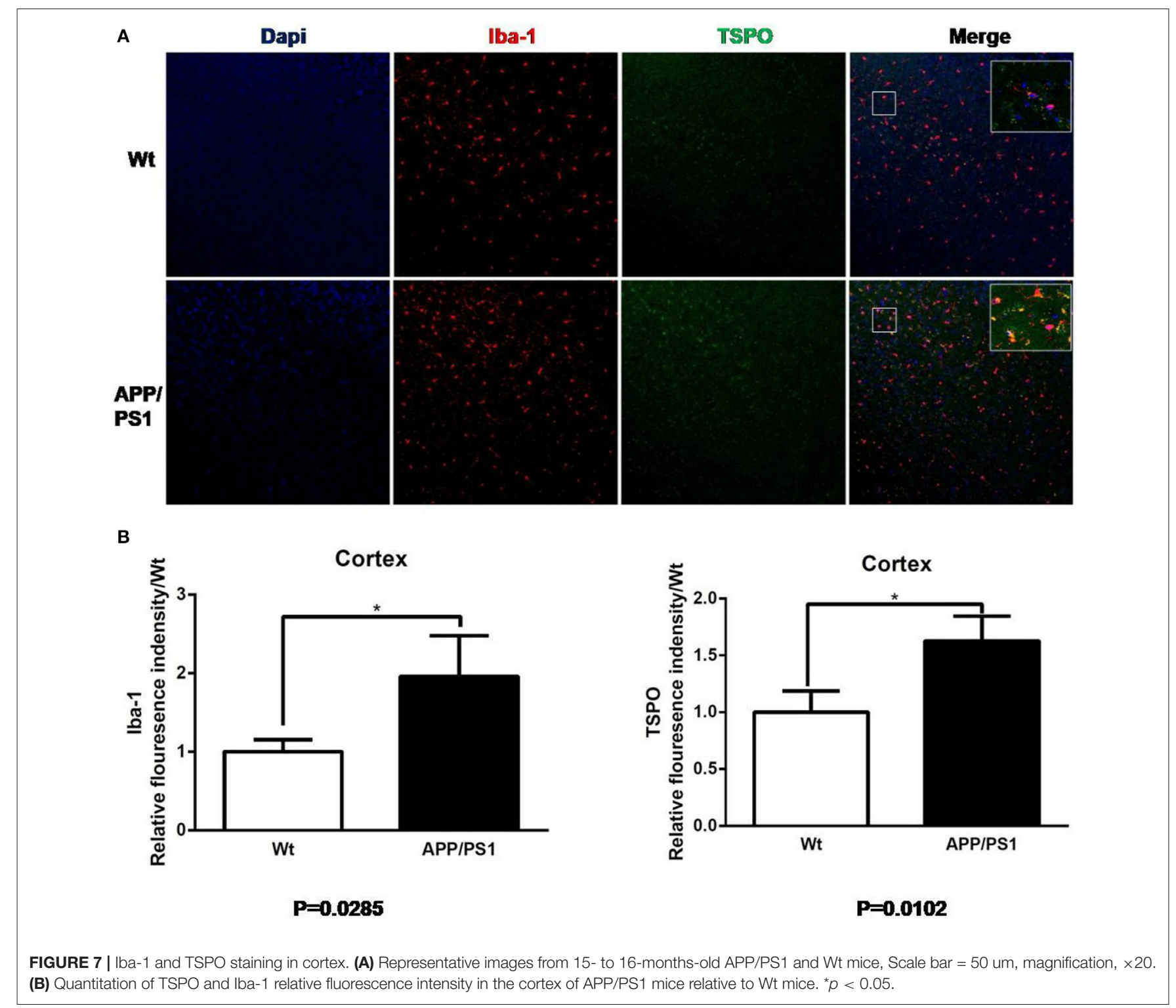

we used PK11195 (1 mg/kg) a known TSPO ligand to block the signals in the cortex and hippocampus of 12-13-months-old APP/PS1 mice before tracer injection (Figure 3B).

Quantitative analysis showed that there was no significant difference of $\left[{ }^{18} \mathrm{~F}\right] \mathrm{DPA} 714$ uptake in our defined VOIs for 6-7months-old (cortex/muscle: $1.63 \pm 0.35$ vs. $1.84 \pm 0.13, P=0.13$; hippocampus/muscle: $1.77 \pm 0.28$ vs. $1.88 \pm 0.17, P=0.24$; $n=8$ ) and $9-10$-months-old (cortex/muscle: $1.51 \pm 0.23$ vs. 1.71 $\pm 0.70, P=0.29$; hippocampus/muscle: $1.76 \pm 0.27$ vs. $1.71 \pm$ $0.33, P=0.40 ; n=8)$ APP/PS1 and Wt mice. We demonstrated the values by $\% \mathrm{ID} / \mathrm{g}$ and selected muscle as a reference region for signal-to-background ratios (Figure 4). There was significantly higher tracer accumulation in our defined VOIs for 12-13 and 15-16-months-old APP/PS1 mice (Figure 5). In the cortex (Figure 6A), we found that the cortex/muscle increased significantly $(\sim 40 \%)$ in APP/PS1 vs. Wt mice at $12-13$ months (cortex/muscle: $2.77 \pm 0.13$ vs. $1.93 \pm 0.32,{ }^{* *} P=0.0014$, $n=8)$ and 15-16 months (cortex/muscle: $2.64 \pm 0.14$ vs. 1.86 $\pm 0.52,{ }^{*} P=0.0159, n=8$ ). In the hippocampus (Figure 6B), the hippocampus/muscle were increased significantly $(\sim 60 \%)$ in APP/PS1 vs. Wt mice at $12-13$ months (hippocampus/muscle: $3.33 \pm 0.10$ vs. $\left.2.10 \pm 0.35,{ }^{* * *} P=0.0008 ; n=8\right)$ and $15-$ 16 months (hippocampus/muscle: $2.89 \pm 0.53$ vs. $1.77 \pm 0.48$, $\left.{ }^{* *} P=0.0050 ; n=8\right)$.

\subsection{Immunofluorescence Staining}

We reaped brain tissues from the 15-16-months-old mice and performed immunofluorescences staining to verify whether the $\left[{ }^{18} \mathrm{~F}\right] \mathrm{DPA} 714$ PET signals were associated with activated microglia and TSPO levels (Figures 7, 8). Figure 7A shows the staining figures in the cortex of Dapi, Iba-1, and TSPO and their merged figure with Wt and APP/PS1 mice. These figures illustrated that compared with Wt mice, APP/PS1 mice had much more activated microglia and a significant higher TSPO level. 


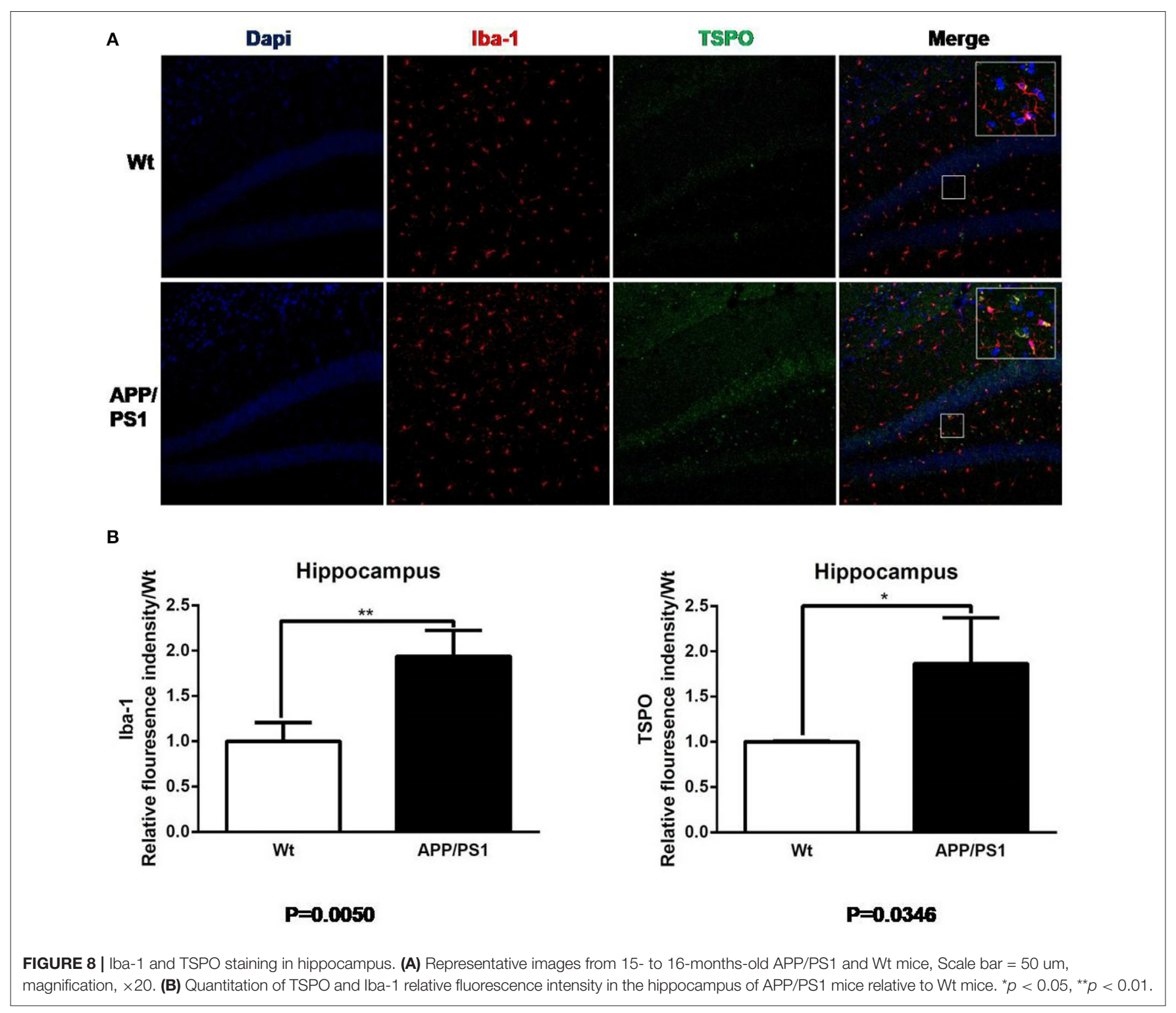

Figure 7B shows the relative fluorescence intensity between Wt and APP/PS1 mice with Iba-1 and TSPO. When the intensity value of $\mathrm{Wt}$ mice for Iba-1 formulated to 1.0 , the relative fluorescence intensity of APP/PS1 mice was 1.87, and the result of $T$ test was ${ }^{*} P=0.0285(n=3 \times 8)$. For TSPO, the relative value was 1.67 and $* P=0.0102(n=3 \times 8)$. The quantitative analysis demonstrated that there was a significant difference between $\mathrm{Wt}$ and APP/PS1 mice in the activated microglia and TSPO level.

Similarly, using the same immunofluorescence staining, Figure 8 also provides the results of the staining figures in chart (A) and the relative fluorescence intensity chart in (B) for the hippocampus. In chart (B), for Iba-1, the relative fluorescence intensity of APP/PS1 mice was 1.95, and the result of the $T$ test was ${ }^{* *} P=0.0050(n=3 \times 8)$. For TSPO, the relative value was 1.81 and ${ }^{*} P=0.0346(n=3 \times 8)$. From the visual analysis in $(A)$ and the quantitative analysis in (B), Figure 8 illustrates that for the hippocampus, APP/PS1 mice also had much more activated microglia and a significantly higher TSPO level than Wt mice. Additionally, in Figure 9, we conducted Pearson correlation analysis and found an obvious correlation between PET signal and Iba-1 relative fluorescence intensity in the cortex $\left(r^{2}=0.76\right)$ and hippocampus $\left(r^{2}=0.88\right)$ and also between PET signal and TSPO relative fluorescence intensity in the cortex $\left(r^{2}=0.87\right)$ and hippocampus $\left(r^{2}=0.67\right)$.

\section{DISCUSSION}

Many studies have shown that TSPO is highly expressed in activated microglia, which makes it a potential target for neuroinflammation imaging. $\left[{ }^{18} \mathrm{~F}\right] \mathrm{DPA} 714$ PET has been used for various neuroinflammation models (Doorduin et al., 2009; Martín et al., 2009; Boutin et al., 2013; Lavisse et al., 2014; Wang et al., 2014). To the best of our knowledge, few tests of $\left[{ }^{18} \mathrm{~F}\right] \mathrm{DPA} 714$ in a well-characterized, age-related APP/PS1 

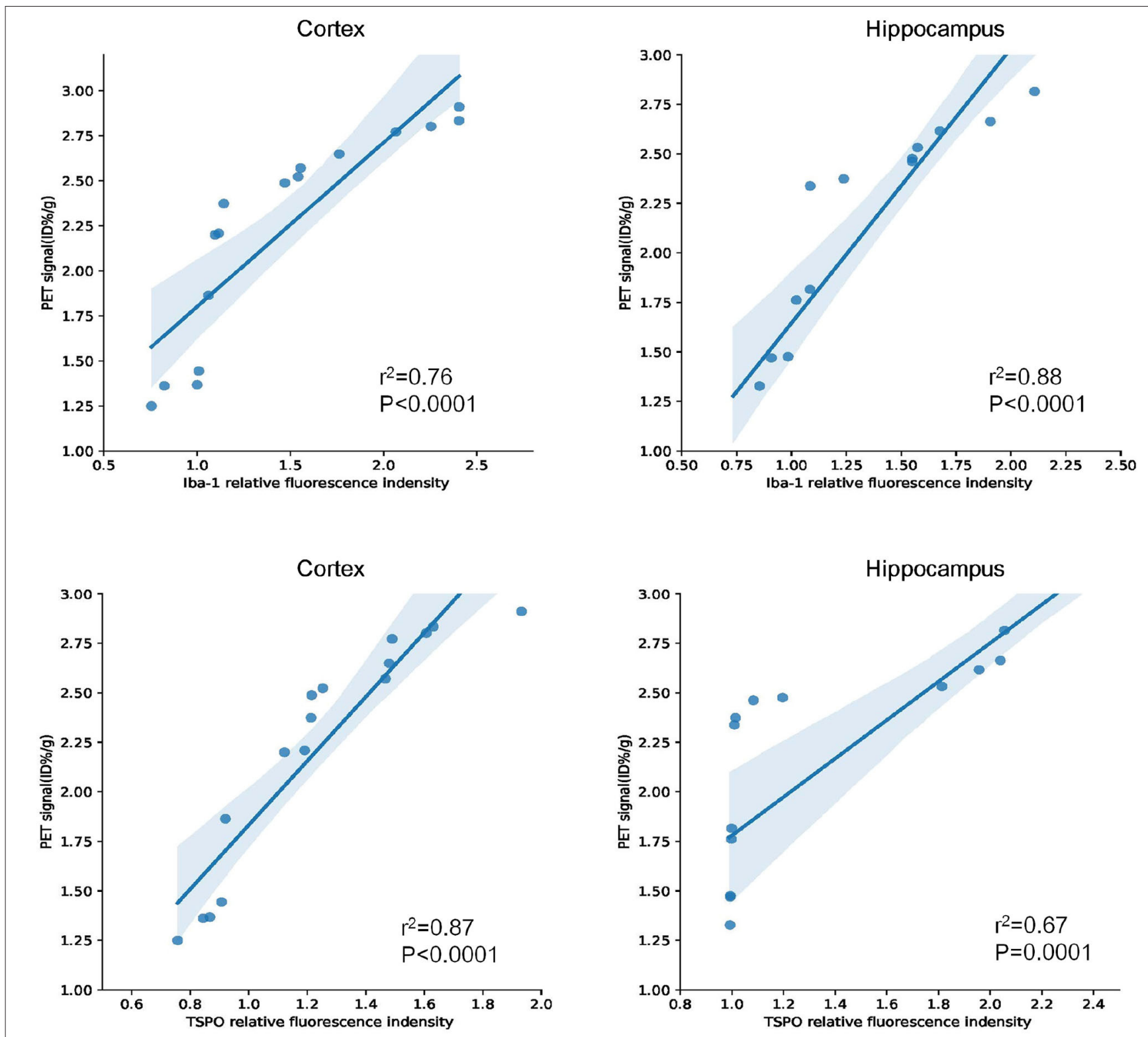

FIGURE 9 | Pearson correlation analysis between Iba-1/TSPO relative fluorescence intensity and PET signal in the cortex and hippocampus for 15-16-months-old APP/PS1 and Wt mice ( $n=8$ /group).

mouse model of AD (Trinchese et al., 2004) have been reported. Our study used $\left[{ }^{18} \mathrm{~F}\right] \mathrm{DPA} 714$ PET to dynamically monitor the neuroinflammatory progression in a mouse model of $\mathrm{AD}$ and to ascertain the possibility of $\left[{ }^{18} \mathrm{~F}\right] \mathrm{DPA} 714$ as an alternative indicator for therapy response in future treatment monitoring studies. In this study, $\left[{ }^{18} \mathrm{~F}\right] \mathrm{DPA} 714$ PET could inspect the activation of microglia and the raised TSPO in the cortex and hippocampus of APP/PS1 mice at 12-13 and 15-16 months old. We confirmed the specificity of $\left[{ }^{18} \mathrm{~F}\right] \mathrm{DPA} 714$ for TSPO in the APP/PS1 mice by blocking experiments using PK11195.

$\mathrm{AD}$ is one of the most common neurodegenerative diseases, which is characterized by memory disorder, aphasia and dementia. Although many scholars have proposed that amyloid deposition and neurofibrillary tangles are related to the disease process of $\mathrm{AD}$, the specific neuropathological changes are still poorly understood, especially for neuroinflammation. In clinical studies, it is relatively difficult to study the neuroinflammatory progression of $\mathrm{AD}$ patients, so, the double transgenic mouse model of APP/PS1 is often chosen to study, so as to simulate neuroinflammation (Yu et al., 2009). Poisnel et al. found that the $\left[{ }^{18} \mathrm{~F}\right]-F D G$ uptake in the brain of APP/PS1 mice were positively correlated with the increase of age. Compared with the age-matched control mice, the uptake of $\left[{ }^{18} \mathrm{~F}\right]-F D G$ in the cortex and hippocampus of 12-months-old APP/PS1 mice 
were higher (Poisnel et al., 2011). André et al. (2012) reported $\left[{ }^{11} \mathrm{C}\right]-\mathrm{PiB}$ uptake in individual brain regions with $\mathrm{A} \beta$ deposition in the APP/PS1 mice as young as 9 months. At present, many PET tracers targeting TSPO, such as $\left[{ }^{11} \mathrm{C}\right] \mathrm{PK} 11195$ (Venneti et al., 2008) and $\left[{ }^{18} \mathrm{~F}\right]$ PBR06 (James et al., 2015) have been utilized in $\mathrm{AD}$ mouse models. In Venneti et al. (2008) study, $\left[{ }^{11} \mathrm{C}\right] \mathrm{PK} 11195$ PET imaging showed that the concentration of tracers in the brain of APP/PS1 mice increased progressively, which related to the histopathological abundance of activated microglia detected by Iba-1 staining. Another study (James et al., 2015) reported that $\left[{ }^{18} \mathrm{~F}\right]$ PBR06, another potential imaging agent for neuroinflammation, gained higher uptake in the cortex and hippocampus of 15-16-months-old $\mathrm{APP}^{L / S}$ mice than $\mathrm{Wt}$, and PET results correlated well with immunostaining. Our study revealed that Iba-1, a marker of activated microglia, is detected in the cortex and hippocampus by immunohistochemistry staining. Additionally, we found that TSPO is highly increased in the cortex and hippocampus of 15-16-months-old APP/PS1 mice, which is co-localized with Iba-1, indicating that TSPO is highly expressed in activated microglia. These results suggest that activated microglia are involved in the disease development of neuroinflammation in AD mice, and elevated TSPO expression can be detected by PET imaging. Thus, activated microglia may be used as an effective target for the treatment of neuroinflammatory damage of $\mathrm{AD}$ in the future.

A non-invasive PET imaging with $\left[{ }^{18} \mathrm{~F}\right] \mathrm{DPA} 714$ for microglia has been used in clinical trials. Arlicot et al. (2012) showed that $\left[{ }^{18} \mathrm{~F}\right] \mathrm{DPA} 714$ is a promising PET radioligand with excellent in vivo bio-distribution and acceptable effective dose estimation in healthy humans. Although the initial clinical studies had shown that $\left[{ }^{18} \mathrm{~F}\right] \mathrm{DPA} 714$ may not be suitable for early diagnosis of $\mathrm{AD}$ patients (Golla et al., 2015), the potential role of $\left[{ }^{18} \mathrm{~F}\right] \mathrm{DPA} 714$ in monitoring TSPO for $\mathrm{AD}$ requires further research. Lorraine et al. (2016) found that microglia are activated in the early stages of $\mathrm{AD}$ and play a protective role in the disease. Subsequent work will involve using $\left[{ }^{18} \mathrm{~F}\right] \mathrm{DPA} 714$ to monitor the anti-inflammatory treatment in an $\mathrm{AD}$ mouse model, which may be helpful in finding a better treatment method for $\mathrm{AD}$.

\section{CONCLUSION}

In this study we optimized the radioactive labeling procedure of $\left[{ }^{18} \mathrm{~F}\right] \mathrm{DPA} 714$ to improve its radiochemical yield and performed a longitudinal PET study that demonstrated increased

\section{REFERENCES}

André, M., Yousefi, B. H., Antje, W., Stefan, P., Sybille, R., Andreas, V., et al. (2012). Small-animal pet imaging of amyloid-beta plaques with $\left[{ }^{11} \mathrm{C}\right] \mathrm{PiB}$ and its multi-modal validation in an APP/PS1 mouse model of Alzheimer's disease. PLoS ONE 7:e31310. doi: 10.1371/journal.pone.0031310

Arlicot, N., Vercouillie, J., Ribeiro, M. J., Tauber, C., Venel, Y., Baulieu, J. L., et al. (2012). Initial evaluation in healthy humans of $\left[{ }^{18} \mathrm{~F}\right] \mathrm{DPA}-714$, a potential pet biomarker for neuroinflammation. Nucl. Med. Biol. 39, 570-578. doi: 10.1016/j.nucmedbio.2011.10.012 inflammation in the brains of APP/PS1 mice compared to Wt mice. Results showed significantly higher signals in our defined VOIs of 12-13 and 15-16 months old APP/PS1 than Wt mice, with levels of TSPO and activated microglia, and no significant differences in $\left[{ }^{18} \mathrm{~F}\right] \mathrm{DPA} 714$ PET signals between younger APP/PS1 and Wt mice. This finding might lead to the selection of mouse age when using $\left[{ }^{18} \mathrm{~F}\right] \mathrm{DPA} 714$ to study the anti-inflammatory treatment of a APP/PS1 mouse model in the future. Our results suggest that $\left[{ }^{18} \mathrm{~F}\right] \mathrm{DPA} 714$, a molecular probe targeting TSPO, has great potential in monitoring microglia activation and neuroinflammation and discovering the best time point of anti-inflammatory therapy for Alzheimer's disease.

\section{DATA AVAILABILITY STATEMENT}

The raw data supporting the conclusions of this article will be made available by the authors, without undue reservation.

\section{ETHICS STATEMENT}

The animal study was reviewed and approved by National Institute of Health Guide for the Care and Use of Laboratory Animals.

\section{AUTHOR CONTRIBUTIONS}

WH together with DP and YW performed the in vivo and immunofluorescence experiments, and JZ, MY, and WB analyzed and interpreted the data and drafted the manuscript and the figures. WH and DP synthesized the radiotracers. CZ, YG, and $\mathrm{FH}$ provided the specific input on the experimental design, data acquisition, and analysis and contributed to manuscript writing. JZ and MY conceived and designed the study and critically revised the manuscript. All authors have seen and agree with the content of the manuscript.

\section{FUNDING}

This project was financially supported by the National Natural Science Foundation of China (NSFC, project 81371625).

\section{ACKNOWLEDGMENTS}

We would like to thank Yuping $\mathrm{Xu}$ for his excellent technical assistance. 
radioligands C-11-DPA-713, F-18-DPA-714, and C-11-PK11195 in a rat model of acute neuroinflammation. J. Nucl. Med. 50, 468-476. doi: 10.2967/jnumed.108.058669

Chen, M. K., and Guilarte, T. R. (2007). Translocator protein 18 kda (TSPO): molecular sensor of brain injury and repair. Pharmacol. Ther. 118, 1-17. doi: 10.1016/j.pharmthera.2007.12.004

Doorduin, J., Klein, H. C., Dierckx, R. A., James, M., Kassiou, M., and Vries, E. F. J. D. (2009). $\left[{ }^{11} \mathrm{C}\right]-\mathrm{DPA}-713$ and $\left[{ }^{18} \mathrm{~F}\right]-\mathrm{DPA}-714$ as new pet tracers for TSPO: a comparison with $\left[{ }^{11} \mathrm{C}\right]-(\mathrm{R})-\mathrm{PK} 11195$ in a rat model of herpes encephalitis. Mol. Imaging Biol. 11, 386-398. doi: 10.1007/s11307-009-0211-6

Garcia-Alloza, M., Robbins, E. M., Zhang-Nunes, S. X., Purcell, S. M., Betensky, R. A., Raju, S., et al. (2007). Characterization of amyloid deposition in the APPSWE/PS1DE9 mouse model of Alzheimer disease. Neurobiol. Dis. 24, 516-524. doi: 10.1016/j.nbd.2006.08.017

Golde, T. E. (2016). Alzheimer disease: host immune defence, amyloidbeta peptide and Alzheimer disease. Nat. Rev. Neurol. 12, 433-434. doi: 10.1038/nrneurol.2016.105

Golla, S. S. V., Boellaard, R., Oikonen, V., Hoffmann, A., van Berckel, B. N. M., Windhorst, A. D., et al. (2015). Quantification of $\left[{ }^{18} \mathrm{~F}\right] \mathrm{DPA}-714$ binding in the human brain: initial studies in healthy controls and Alzheimer's disease patients. J. Cereb. Blood Flow Metab. 35, 766-772. doi: 10.1038/jcbfm.2014.261

James, M. L., Belichenko, N. P., Nguyen, T. V. V., Andrews, L. E., and Chin, F. T. (2015). PET imaging of translocator protein $(18 \mathrm{kda})$ in a mouse model of Alzheimer's disease using ${ }^{18}$ F-PBR06. J. Nucl. Med. 56, 311-316. doi: 10.2967/jnumed.114.141648

James, M. L., Fulton, R. R., Vercoullie, J., Henderson, D. J., Garreau, L., Chalon, S., et al. (2008). DPA-714, a new translocator protein-specific ligand: synthesis, radiofluorination, and pharmacologic characterization. J. Nucl. Med. 49, 814-822. doi: 10.2967/jnumed.107.046151

James, M. L., and Gambhir, S. S. (2012). A molecular imaging primer: modalities, imaging agents, and applications. Physiol. Rev. 92, 897-965. doi: 10.1152/physrev.00049.2010

Jankowsky, J. L., Fadale, D. J., Anderson, J., Xu, G. M., Gonzales, V., Jenkins, N. A., et al. (2003). Mutant presenilins specifically elevate the levels of the 42 residue beta-amyloid peptide in vivo: evidence for augmentation of a 42 -specific gamma secretase. Hum. Mol. Genet. 13, 159-170. doi: 10.1093/hmg/ddh019

Keller, T., López-Picón, F. R., Krzyczmonik, A., Forsback, S., Kirjavainen, A. K., Takkinen, J. S., et al. (2018). $\left[{ }^{18} \mathrm{~F}\right] \mathrm{F}-\mathrm{DPA}$ for the detection of activated microglia in a mouse model of alzheimer's disease. Nucl. Med. Biol. 67, 1-9. doi: 10.1016/j.nucmedbio.2018.09.001

Lavisse, S., K, I., Jan, C., Peyronneau, M.-A., Petit, F., S, G., et al. (2014). [ ${ }^{18}$ F]DPA714 PET imaging of translocator protein tspo $(18 \mathrm{kda})$ in the normal and excitotoxically-lesioned nonhuman primate brain. Eur. J. Nucl. Medi. Mol. Imaging 42, 478-494. doi: 10.1007/s00259-014-2962-9

Li, Y., Tan, M. S., Jiang, T., and Tan, L. (2014). Microglia in Alzheimer's disease. Biomed. Rese. Int. 2014:437483. doi: 10.1155/2014/437483

Lorraine, H., Julien, L., Guillaume, D., Claire, L., Mickael, L., A., et al. (2016). Early and protective microglial activation in Alzheimer's disease: a prospective study using ${ }^{18}$ F-DPA-714 PET imaging. Brain 139, 1252-1264. doi: 10.1093/brain/aww017

Martín, A., Boisgard, R., Thézé, B., Camp, N. V., and Tavitian, B. (2009). Evaluation of the PBR/TSPO radioligand $\left[{ }^{18} \mathrm{~F}\right] \mathrm{DPA}-714$ in a rat model of focal cerebral ischemia. J. Cereb. Blood Flow Metab. 30, 230-241. doi: 10.1038/jcbfm. 2009.205
Peyronneau, M.-A., Saba, W., Goutal, S., Damont, A., Dollé, F., Kassiou, M., et al. (2012). Metabolism and quantification of [F-18] DPA-714, a new TSPO positron emission tomography radioligand. Drug Metab. Dispos. 41, 122-131. doi: $10.1124 / \mathrm{dmd} .112 .046342$

Poisnel, G., Hérard, A. S., Tayara, N. E. T. E., Bourrin, E., and Dhenain, M. (2011). Increased regional cerebral glucose uptake in an APP/PS1 model of Alzheimer's disease. Neurobiol. Aging 33, 1995-2005. doi: 10.1016/j.neurobiolaging.2011.09.026

Politis, M., Su, P., and Piccini, P. (2012). Imaging of microglia in patients with neurodegenerative disorders. Front. Pharmacol. 3:96. doi: 10.3389/fphar.2012.00096

Rupprecht, R., Papadopoulos, V., Rammes, G., Baghai, T. C., Fan, J., and Akula, N. (2012). Translocator protein (18 kda) (TSPO) as a therapeutic target for neurological and psychiatric disorders. Nat. Rev. Drug Discov. 9, 971-988. doi: $10.1038 / \mathrm{nrd} 3295$

Takkinen, J. S., López-Picón, F. R., Majidi, R. A., Eskola, O., and HaaparantaSolin, M. (2016). Brain energy metabolism and neuroinflammation in ageing APP/PS1-21 mice using longitudinal 18 F-FDG and 18 FDPA-714 PET imaging. J. Cereb. Blood Flow Metab. 37, 2870-2882. doi: 10.1177/0271678X16677990

Trinchese, F., Liu, S., Battaglia, F., Walter, S., Mathews, P. M., and Arancio, O. (2004). Progressive age-related development of Alzheimer-like pathology in APP/PS1 mice. Ann. Neurol. 55, 801-814. doi: 10.1002/ana.20101

Venneti, S., Lopresti, B. J., Wang, G., Hamilton, R. L., and Wiley, C. A. (2008). Pk11195 labels activated microglia in Alzheimer's disease and in vivo in a mouse model using pet. Neurobiol. Aging 30, 1217-1226. doi: 10.1016/j.neurobiolaging.2007.11.005

Vivash, L., and O'Brien, T. J. (2016). Imaging microglial activation with TSPO PET: lighting up neurologic diseases? J. Nucl. Med. 57, 165-168. doi: 10.2967/jnumed.114.141713

Wang, Y., Yue, X., Kiesewetter, D. O., Niu, G., Teng, G., and Chen, X. (2014). PET imaging of neuroinflammation in a rat traumatic brain injury model with radiolabeled TSPO ligand DPA-714. Eur. J. Nucl. Med. Mol. Imaging 41, 1440-1449. doi: 10.1007/s00259-014-2727-5

Xiong, H., Callaghan, D., Wodzinska, J., Xu, J., Premyslova, M., Liu, Q. Y., et al. (2011). Biochemical and behavioral characterization of the double transgenic mouse model (APPSWE/PS1DE9) of Alzheimer's disease. Neurosci. Bull. 27, 221-232. doi: 10.1007/s12264-011-1015-7

Yu, Y., He, J., Zhang, Y., Luo, H., Zhu, S. S., Yang, Y., et al. (2009). Increased hippocampal neurogenesis in the progressive stage of Alzheimer's disease phenotype in an APP/PS1 double transgenic mouse model. Hippocampus 19, 1247-1253. doi: 10.1002/hipo.20587

Conflict of Interest: The authors declare that the research was conducted in the absence of any commercial or financial relationships that could be construed as a potential conflict of interest.

Copyright (C) $2020 \mathrm{Hu}$, Pan, Wang, Bao, Zuo, Guan, Hua, Yang and Zhao. This is an open-access article distributed under the terms of the Creative Commons Attribution License (CC BY). The use, distribution or reproduction in other forums is permitted, provided the original author(s) and the copyright owner(s) are credited and that the original publication in this journal is cited, in accordance with accepted academic practice. No use, distribution or reproduction is permitted which does not comply with these terms. 